\title{
On metrics for measuring scanpath similarity
}

\author{
Ramin Fahimi $^{1}$ (D) Neil D. B. Bruce ${ }^{2}$ \\ Published online: 10 August 2020 \\ (C) The Psychonomic Society, Inc. 2020
}

\begin{abstract}
Saliency and visual attention have been studied in a computational context for decades, mostly in the capacity of predicting spatial topographical saliency maps or simulated heatmaps. Spatial selection by an attentive mechanism is, however, inherently a sequential sampling process in humans. There have been recent efforts in analyzing and modeling scanpaths, however, there is as of yet no universal agreement on what metrics should be applied to measure scanpath similarity or the quality of a predicted scanpath from a computational model. Many similarity measures have been suggested in different contexts and little is known about their behavior or properties. This paper presents in one place a review of these metrics, axiomatic analysis of gaze metrics for scanpaths, and careful analysis of the discriminative power of different metrics in order to provide a roadmap for further future analysis. This is accompanied by experimentation based on classic modeling strategies for simulating sequential selection from traditional representations of saliency, and deep neural networks that produce sequences by construction. Experiments provide strong support for the necessity of sequential analysis of attention and support for certain metrics including a family of metrics introduced in this paper motivated by the notion of scanpath plausibility.
\end{abstract}

Keywords Saliency $\cdot$ Visual attention $\cdot$ Eye movement $\cdot$ Scanpath

\section{General introduction}

Human and animal brains have significant yet limited computational power, especially when considering the amount of sensory data available to them. Given this limitation, the brain appears to have evolved to provide an efficient solution to actively control the stream of information. Attention is the process of selectively concentrating on some portion of available information, at the expense of ignoring other perceivable parts. It is a combination of behavioral and cognitive processes and it may be defined subjectively or objectively. It has been widely researched in cognitive and perceptual psychology andregardless of discipline, the usage has been identified to be critical to Information Reduction and filtering.

Ramin Fahimi

fahimir@myumanitoba.ca

Neil D. B. Bruce

brucen@uoguelph.ca

1 Computer Science, University of Manitoba, Winnipeg, Canada

2 School of Computer Science, University of Guelph, Guelph, Canada
Visual attention is a form of attention that allows humans and animals to control and allocate their limited visual processing resources. The form of control varies across species. Attention is a complex process that may also include physical actions like eye or head movements. In humans, it has been posited that more than $60 \%$ of the brain is involved in processing visual data; a high proportion of activated capacity. It is not that there is too much information, the problem is that each component of each stimulus can be matched to many different objects or types of scenes in our memory resulting in an explosion of potential interpretations. In general, attention is a crucial mechanism for animal and human survival.

Sequences of successive eye movements during scene exploration are called visual scanpaths. The human eye reveals a rapid drop-off in visual acuity and most neurons are positioned in front of the fovea. This means that humans have to move their gaze to effectively place a region of interest (ROI) in front of their fovea where they have the highest visual acuity. During sequences of eye movements, the eyes tend to pause briefly on certain locations that are known as fixations. Fixations are followed by saccades, which involve rapid changes in eye position driven by a ballistic action that rapidly accelerates the rotation of the eyes to land at a new target (and with some imprecision). 
Extracting visual information takes place during the fixation period and the whole process enables humans to do finegrained analysis of the scene. Not all of the spatial locations will be viewed and the selective process and its ordering become important. Other parameters are also involved in this. Visual inhibition of return (IOR) facilitates foraging by discouraging re-examination of recently fixated locations (Samuel \& Kat, 2003). Due to the limited capacity of visual working memory (VWM), effects of IOR gradually fade over time, which allows the possibility to re-fixate previously attended regions.

Saliency and visual attention have been studied in a computational context for decades. Much of the emphasis of studies has been focused on the notion of saliency, defined by Koch and Ullman (1985) as a two-dimensional topographic representation of conspicuity for every location (pixel) in the image. This has been used to characterize the spatial density of visual fixations (Bruce et al., 2016), objects of interest in a scene (Islam et al., 2018; Islam et al., 2017), the degree of objectness (Kuo et al., 2015) of pixels or bounded regions and other less common definitions. Other efforts have also sought to attach task to this, examining these densities from a top-down perspective (Adeli \& Zelinsky, 2018). Moreover, the notion of attention has become increasingly prevalent in the context of deeplearning wherein recurrent mechanisms or reinforcement learning drive the gradual solution to a problem, in some instances involving spatial selection (Xu et al., 2015).

While this problem has been studied in some detail, the overwhelming majority of work in this domain considers a static problem wherein the distribution of gaze, attention or salience is marked by its spatial distribution. However, spatial selection by an attentive mechanism is inherently a sequential sampling process in humans and many artificial vision systems. This evidently gains additional importance as sequential attention mechanisms in artificial vision systems are becoming increasingly prevalent. There may be a strong bias to examine certain parts of a scene right away before moving on to other interesting regions. On the other hand, there may be several equally interesting regions of a scene and no particular order. This is a characteristic that most modeling and analysis to date has failed to address in detail. While some efforts have been devoted to analyzing sequential models of fixation, the case may be made (as it is in this paper) that existing metrics fall short in adequately capturing model performance, and similarities and differences among sequences of selected regions. Moreover, it is revealed that certain metrics are far more discriminative than others, and become even more discriminative when viewed through the lens of scanpath plausibility, an extension to existing metrics we propose in this work.

\section{Motivation and contributions}

Established algorithms for saliency prediction have been improving over the years in predicting patterns derived from gaze data. However, in the vast majority of cases, they have been tested with datasets containing few salient objects and more importantly, the focus of this analysis and measurement of success has been based on 2D topographical representations of what is salient. Moreover, with more crowded and occluded scenes including many natural images, the attentive behavior of humans varies significantly from one person to another. Given that this is not about right or wrong patterns of viewing, sequential analysis could be a more insightful avenue for investigation.

In general, simulating and modeling the human visual system has the advantage that it results in human-like behavior. This is beneficial for systems that need to interact with humans in a natural manner. Seeking simple solutions to derive a sequence of fixation points while exploring a scene could also help attentive models produce higher accuracy for their specific task. High-level strategies for optimal information gathering are key to the field of computer vision. Most applications in computer vision function primarily in a passive way. Meanwhile, active control over the acquisition of image data is fundamental to efficient development of robust and general computer vision solutions for unconstrained environments. The advent of deep learning has been something of a revolution in machine vision capabilities and has brought a significant boost in the capabilities and performance of solutions. However, even in this domain there is no escaping the large amount of computing power necessary to adequately treat input especially if the desire is a solution that is possible to run in real time. Incorporating attention in models could help converge to a better solution. This also raises the question of what value can be gained, or what can be understood in comparing the attentive policy of models built on machine learning models for specific tasks with humans.

Motivated by the aforementioned observations, we seek to revisit the space of metrics currently used in the domain of analyzing gaze trajectories, viewing patterns and saliency in order to arrive at a consensus on intuitive interpretations of inter-sequence distances, and also towards redefining metrics that produce meaningful and significant contrast among observations. In the balance of this paper, we present metrics and experimentation that advocates for alternative metrics to any appearing in the literature deemed ScanPath Plausibility (SPP). This is tested in considering a wide variety of extant saliency models coupled with a selection mechanism.

Contributions of this paper are therefore as follows: 
- A detailed analysis and review of scanpath measures. This work carries empirical experiments on quantification of metric behavior rather than mainly discussing metrics from a theoretical standpoint.

- Revealing strengths and weaknesses of similarity measures from both an axiomatic and empirical standpoint along with recommendations for analysis.

- Comparative studies based on information in a sequence and showing the wealth of information comparing to a static representation.

\section{Scanpath analysis approaches}

Various measures have been proposed to compare two scanpaths. Some of them behave like a similarity measure and some like a dissimilarity measure. The simplest form of these metrics are direct measures based on Euclidean distance. String-based measures work by quantizing the spatial landscape into different bins (Cristino et al., 2010; Duchowski et al., 2010) and use of string alignment metrics. Vector-based methods try to geometrically align scanpaths (e.g., MultiMatch Dewhurst et al. (2012)) and do not necessarily provide a measure of models of visual exploration that is readily interpretable. More sophisticated curve similarity measures, time-series metrics and recurrence analysis are less common but they may be more discriminative in revealing differences between viewing patterns or computational models. Table 1 highlights commonly used metrics in this area. A useful metric should consider position, ordinal characteristics and perhaps duration and dynamics of fixations.
Given two scanpaths in two-dimensional Cartesian coordinates, namely $P=\left(p_{1}, p_{2}, \ldots, p_{N}\right), Q=\left(q_{1}, q_{2}, \ldots, q_{M}\right)$ with lengths $\mathrm{N}$ and $\mathrm{M}$, respectively, recorded while observing a stimuli or image with a width of $\mathrm{W}$ and height of $\mathrm{H}$, this discussion reviews metrics that have been introduced for comparing sequences based on various criteria.

\section{Direct measures}

\section{Euclidean distance}

Euclidean distance or straight-line distance is one of the initial metrics that was used in comparing scanpaths. The distance can be calculated as the sum of the distances between fixation pairs each with two-dimensional Cartesian coordinates $\left(P_{i}^{X}, P_{i}^{Y}\right)$. This only works for sequences of fixations with equal lengths, hence in general the shared length should be considered:

$$
D_{E U C}(P, Q)=\sum_{i=1}^{\min (N, M)} \sqrt{\left(P_{i}^{X}-Q_{i}^{X}\right)^{2}+\left(P_{i}^{Y}-Q_{i}^{Y}\right)^{2}}
$$

\section{Mannan distance}

Mannan et al. (1996) is a direct measure that considers exact position rather than quantizing AOIs. Let $d(M x N)$ be the two-dimensional pair-wise Euclidean distance of

Table 1 Common metrics for evaluation of Scanpaths. Type of scanpath measures include similarity (S), dissimilarity (D), and quantization includes Grid (G), Grid-Temporal (GT) and Radius (R) measures

\begin{tabular}{|c|c|c|c|c|c|c|c|}
\hline & Metric & Abrv & Type & Qnt. & Position & Order & Duration \\
\hline 1 & Euclidean distance & EUC & $\mathrm{D}$ & - & $\checkmark$ & $\mathrm{X}$ & $\mathrm{X}$ \\
\hline 2 & Mannan distance (Mannan et al., 1996) & MAN & $\mathrm{D}$ & - & $\checkmark$ & $\mathrm{X}$ & $\mathrm{X}$ \\
\hline 3 & Eyeanalysis (Mathôt et al., 2012) & EYE & $\mathrm{D}$ & - & $\checkmark$ & $\mathrm{X}$ & $\mathrm{X}$ \\
\hline 4 & Levenshtein distance (Privitera \& Stark, 2000) & LEV & $\mathrm{D}$ & $\mathrm{G}$ & $\checkmark$ & $\checkmark$ & $\mathrm{X}$ \\
\hline 5 & ScanMatch (Cristino et al., 2010) & SMT & $\mathrm{S}$ & GT & $\checkmark$ & $\checkmark$ & $\checkmark$ \\
\hline 6 & Hausdorff distance (Huttenlocher et al., 1993) & HAU & $\mathrm{D}$ & - & $\checkmark$ & $\mathrm{X}$ & $\mathrm{X}$ \\
\hline 7 & Frechet distance (Eiter \& Mannila, 1994) & FRE & $\mathrm{D}$ & - & $\checkmark$ & $\checkmark$ & $\mathrm{X}$ \\
\hline 8 & Dynamic time warp (Berndt, 1994) & DTW & $\mathrm{D}$ & - & $\checkmark$ & $\checkmark$ & $\mathrm{X}$ \\
\hline 9 & Time delay embedding (Wang et al., 2011) & TDE & $\mathrm{D}$ & - & $\checkmark$ & $\mathrm{X}$ & $\mathrm{X}$ \\
\hline 10 & MultiMatch shape (Dewhurst et al., 2012) & MM_S & $\mathrm{S}$ & - & $\mathrm{X}$ & $\mathrm{X}$ & $\mathrm{X}$ \\
\hline 11 & MultiMatch direction(Dewhurst et al., 2012) & MM_A & $\mathrm{S}$ & - & $\mathrm{X}$ & $\mathrm{X}$ & $\mathrm{X}$ \\
\hline 12 & MultiMatch length (Dewhurst et al., 2012) & MM_L & $\mathrm{S}$ & - & $\mathrm{X}$ & $\mathrm{X}$ & $\mathrm{X}$ \\
\hline 13 & MultiMatch position (Dewhurst et al., 2012) & MM_P & $\mathrm{S}$ & - & $\checkmark$ & $\mathrm{X}$ & $\mathrm{X}$ \\
\hline 14 & MultiMatch duration (Dewhurst et al., 2012) & MM_D & $\mathrm{S}$ & - & $\mathrm{X}$ & $\mathrm{X}$ & $\checkmark$ \\
\hline 15 & Recurrence (Anderson et al., 2013b) & REC & - & $\mathrm{R}$ & $\checkmark$ & $\mathrm{X}$ & $\mathrm{X}$ \\
\hline 16 & Determinism (Anderson et al., 2013b) & DET & - & $\mathrm{R}$ & $\mathrm{X}$ & $\checkmark$ & $\mathrm{X}$ \\
\hline 17 & Laminarity (Anderson et al., 2013b) & LAM & - & $\mathrm{R}$ & $\mathrm{X}$ & $\mathrm{X}$ & $\mathrm{X}$ \\
\hline 18 & Corm (Anderson et al., 2013b) & COR & - & $\mathrm{R}$ & $\mathrm{X}$ & $\mathrm{X}$ & $\mathrm{X}$ \\
\hline
\end{tabular}


every fixation in $P$ with all other fixations in $Q . D_{P, Q}$ is equal to the weighted mean of minimum distances for each fixation in $P$ when compared with all fixations in $Q$. This is usually normalized by resolution. As outlined in Anderson et al. (2015), similarity index (Is) is the Mannan distance normalized with the Mannan distance of two random sequence $D_{r}$, as noted in Eq. 3. Use of absolute positions is intuitive and also might make it possible to use raw data from eye trackers. However, as each fixation is associated with its nearest neighbor, the ordinal information is mostly ignored and it softens the impact of individual fixations. This makes it difficult to retain and consider the sequential nature of information.

$$
\begin{aligned}
& D^{2}(P, Q)=\frac{M * \sum_{i=1}^{N} \min d_{P i}^{2}+N * \sum_{j=1}^{M} \min d_{Q j}^{2}}{2 * N * M *\left(W^{2}+H^{2}\right)} \\
& I s=\left[1-\frac{D_{P, Q}}{D_{r}}\right] * 100
\end{aligned}
$$

\section{Eyeanalysis}

A few alternative approaches (Foulsham \& Underwood, 2008; Henderson et al., 2007; Tatler et al., 2005) have been proposed to rectify some of the shortcomings of Mannan distance. "Eyeanalysis" (Mathôt et al., 2012) proposes double mapping instead of mapping one fixation to one other fixation. This provides the lowest overall position variability but still may lead to several points in one scanpath being mapped to just one in the other. There exists also the possibility of including the duration of fixation. It is not a direct measure for ordering of fixations but allows the temporal characteristics of the sequence to be taken into account to a limited degree.

$$
D_{E Y E}=\frac{\left(\sum_{j=1}^{N} \min d_{P j}^{2}+\sum_{i=1}^{M} \min d_{Q i}^{2}\right)}{\max (N, M)}
$$

\section{String-based metrics}

\section{Levenshtein similarity (Edit distance)}

The use of Levenshtein similarity a.k.a edit distance (Privitera \& Stark, 2000) for the purpose of comparing scanpaths dates back to Noton and Stark (1971) as one of the very first metrics used in literature. The Levenshtein algorithm originally compares two sets of DNA strings $A\left(a_{1}, a_{2},, . . a_{a}\right), B\left(b_{1}, b_{2}, . . b_{b}\right)$ with lengths $a$ and $b$ based on the minimum number of insertions, deletions, and substitutions to produce two identical sequences.

To be able to use this algorithm for scanpath comparison, fixation coordinates need to be converted to a string. As originally used in Noton's studies (Noton \& Stark, 1971), a static grid is overlaid on the stimulus to discretize areas of interest; Each grid cell is assigned an alphabetic character. Following this, a series of fixations can be represented using a string of characters and the Levenshtein algorithm can be used to compare strings representing them. As powerful as it may seem in considering ordinal information, there are various factors related to this algorithm and other string-based metrics that aren't fully taken into account. For instance, the spatial position of fixations is not well represented and this can have consequences for the similarity metric. Two fixations that are spatially close, can land on different bins of the grid which could lead to an inaccurate distance. Similarly, fixations occupying the same bins are often grouped and represented with one letter. This has the effect of ignoring local differences in eye movement control and ongoing cognitive process. In general, the Levenshtein algorithm is blind to the semantic contents of an image and cannot distinguish differences in substitutions of fixations from different regions. Moreover, duration of fixations is completely ignored in this algorithm.

\section{ScanMatch}

ScanMatch can be seen as improved version of Levenshtein distance. It has recently emerged in the literature (Cristino et al., 2010) and has been used in numbers of studies (Pellicano et al., 2011; Sharafi et al., 2015; Foulsham et al., 2012). ScanMatch tries to solve some of the shortcomings of Levenshtein distance in considering semantic information and duration of fixations. Levenshtein distance treats all differences between strings equally. The edit cost of substituting, inserting, and deleting is the same in all cases, and this results in being blind about information lying within other areas of interest. The advantage of this metric comes from use of the Needleman-Wunsch pair-wise algorithm (Needleman \& Wunsch, 1970) which aligns two strings with one another to maximize a similarity score. The Needleman-Wunsch algorithm uses a substitution matrix containing all of the pairings in two strings. This makes it possible to weight the substitution operation for various areas of interest (Fig. 1).

ScanMatch metrics follow similar pre-processing steps to Levenshtein distance for converting scanpaths to a sequence of strings. Following this, the objective is to fill a comparison matrix representing two strings on different axes based on the substitution matrix. The algorithms search for an optimal path through this matrix from top-left to bottom-right to calculate the similarity score and subsequently the normalized score subject to the length of the sequence provides the final resulting score. While ScanMatch has the advantage of not being blind to semantic content, similar to Levenshtein distance, it still suffers from 

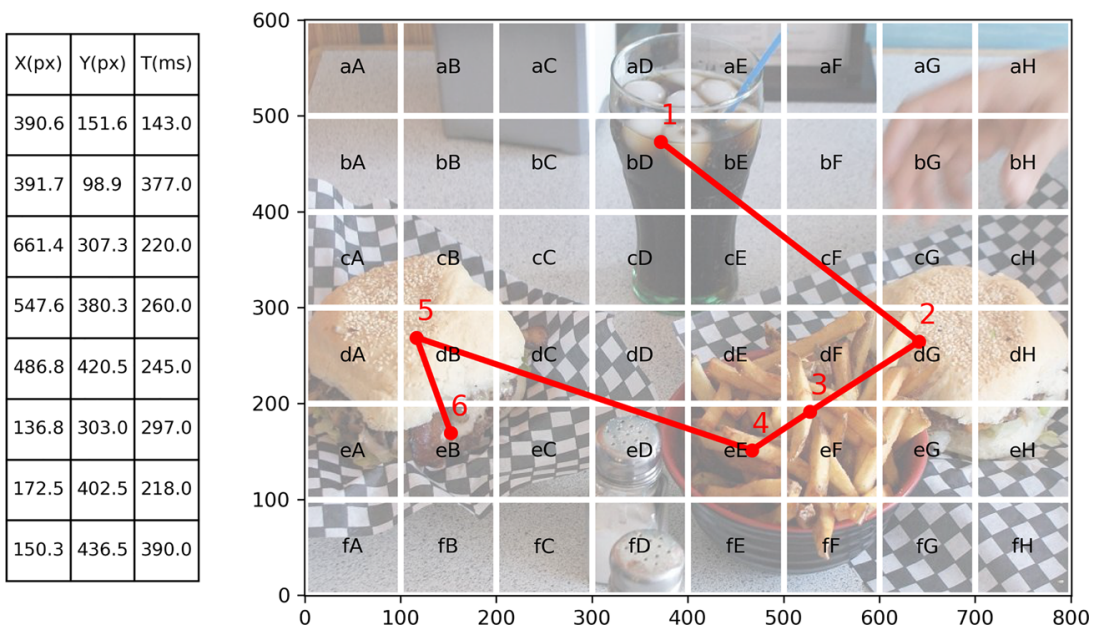

Fig. 1 a A sample scanpath b Visualization of Scanpath in (a) and pre-processing stage for transforming scanpaths to strings used in ScanMatch algorithm. "bDdGeFeEdBeB" without considering duration and "bDdGdGdGeFeFeEeEdBdBeBeB" when T = 100ms

problems arising as the result of discretizing the AOIs and ignoring the exact positions of fixations.

One interesting feature that can be included in this process is the duration of fixation points. The duration of a fixation can be quantized according to a fixed temporal bin and for as long as the fixation stays, with the character/string representing the AOI repeated in the overall sequence. For instance, if a fixation takes $t \mathrm{~ms}$ and the bin is $\mathrm{T} \mathrm{ms}$, the character representing the bin will be repeated $\left\lceil\frac{t}{T}\right\rceil$ times.

It is worth mentioning that throughout this paper we considered a grid of $12 \times 8$ for experiments.

\section{Curve similarity}

\section{Hausdorff distance}

Hausdorff distance (Huttenlocher et al., 1993) represents the degree of mismatch between two sets by measuring the farthest distance from one set to the other. Hausdorff is mostly used for measuring the distance between two curves in space yet it does not consider temporal information. Hausdorff measures how far is the maximum distance among the mapping between sets based on minimum distance. Given two scanpaths P and Q, Hausdorff distance is defined as follows:

$$
\begin{aligned}
& D_{H A U}(P, Q)=\max (h(P, Q), h(Q, P)) \\
& h(A, B)=\operatorname{maxmin}_{a \in P \in Q}\|a-b\|
\end{aligned}
$$

\section{Discrete Frechet distance}

The Frechet distance (Aronov et al., 2006; Eiter \& Mannila, 1994) is a measure of similarity between two curves. Unlike Hausdorff, Frechet accounts for position and ordering of fixations. Consider a dog walking along one curve and the owner walking along the other curve connected by a leash. With both walking continuously from their respective starting points, and varying their speed but not backtracking, the Frechet distance between the two curves is the length of the shortest leash sufficient for traversing the curves:

$$
D_{F R E(A, B)}=\inf _{\alpha, \beta} \max _{t \in[0,1]}\{d(P(\alpha(t)), Q(\beta(t)))\}
$$
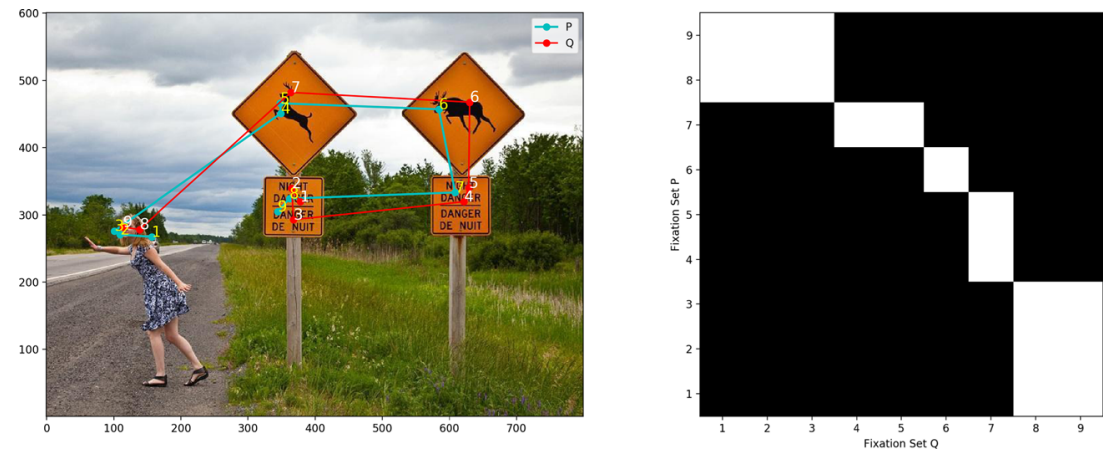

Fig. 2 Scanpath $\mathrm{P}$ and $\mathrm{Q}$ visualized on left along with their respective recurrence matrix based on Euclidean distance with $\rho=2 * 24$ (visual angle) on the right. Image size is $600 \times 800$ 


\section{Time-series analysis}

\section{Dynamic time warping}

Dynamic time warping (Berndt, 1994) can compare two time-series with varying (and differing) lengths. DTW has different variations. Given two sequences with length $\mathrm{N}$ and $\mathrm{M}$, the most common DTW builds an NxM matrix of respective Euclidean distances (D). Following this, the algorithm tries to find an optimal path from $(0,0)$ to $(\mathrm{N}, \mathrm{M})$ to match both sequences while preserving three conditions: boundary, continuity, and monotonicity to make sure that the path respects time. Boundary guarantees that the alignment does not only partially consider one of the sequences. Continuity checks for not jumping in time and missing important features and lastly Monotonicity guarantees always moving forward in time. The optimal solution is the minimum among all the paths in order to get from $(0,0)$ to $(\mathrm{N}, \mathrm{M})$ as described in Algorithm 1.

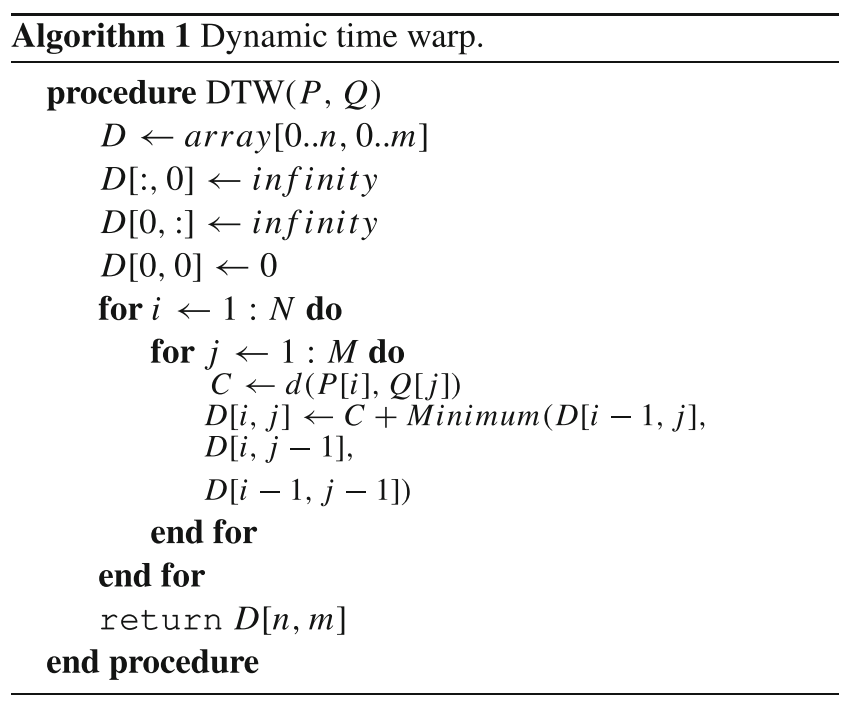

\section{Time delay embedding}

Time delay embedding (Sauer et al., 1991) has been widely used in the study of dynamical systems and was introduced for scanpath comparison by Wang et al. (2011) et al. TDE tries to consider the ordering by creating consecutive subsamples. P and Q go through the same pre-processing step by breaking each into consecutive samples with length $\mathrm{K}$. The final output comes from calculating mean or maximum (Hausdorff) of pair-wise Euclidean distance between subsamples from each scanpath. The parameter $\mathrm{K}$ plays an important role in handling spatial and ordinal noise.

\section{Vector-based metrics}

\section{MultiMatch}

Multimatch (Meur, 2015) is one of the frequently used metrics in the literature. It calculates the distance based on five fundamental characteristics of saccades between fixations: shape, length, direction, position, and duration. The main advantage of the MultiMatch method is that it provides several measures to choose from for assessing scanpath similarity with each capturing a unique component of scanpath similarity. Given the multiplicity of measures, it remains difficult to assess which measure, or which set of measures, is most applicable in a given scenario. Furthermore, because each scanpath is initially simplified it is also not clear how robust each measure is to scanpath variations (Anderson et al., 2015).

The algorithm follows a pre-processing stage in which scanpaths are simplified in order to cluster fixations and saccades that are close together or represent local scanning. It starts with direction and continues with length and for as long as there are consecutive saccades that are smaller than a fixed threshold, they will be either merged or removed. There are some precautionary measures that need to be taken into account when simplifying scanpaths. Merging saccades with small changes in direction while they are large in terms of amplitude might cause a loss of some important information. Following the pre-processing step, scanpaths will be aligned based on their shape using Dijkstra's algorithm. Alignment could happen based on any of the other four parameters but it is common to do so based on shape. This alignment reduces the comparison sensitivity to small temporal or spatio-temporal variations, and allows the algorithm to find the best possible match between the pair of scanpaths.

MultiMatch Vector similarity is computed as the vector difference between aligned saccade pairs. The Length similarity is computed as the absolute difference in the amplitude of aligned saccade vectors and the position similarity is computed as the Euclidean distances between aligned fixations. All three of these measurements are normalized by the screen diagonal and averaged over scanpaths. The Direction Similarity is computed as the angular difference between aligned saccades, normalized by $\pi$ and averaged over scanpaths. Finally, the duration similarity is computed as the absolute difference in fixation durations of aligned fixations, normalized by the maximum duration and averaged over scanpaths. 


\section{Recurrence analysis}

\section{Recurrence, determinism, laminarity, CORM}

Recurrence quantification analysis (RQA) has been successfully used for describing dynamic systems that are too complex to be characterized adequately by standard methods in time series analysis. RQA was initially conceptualized as a method for capturing the temporal dynamics of a single scanpath and it was later expanded to CRQA for scanpath comparison by Anderson et al. (2013a). Despite the simplicity in definition, this family of metrics has a clear interpretation and intuitive meaning which makes them able to capture global and local temporal characteristics of a sequence by a small number of RQA measures. All four metrics are somehow based on the recurrence matrix $r$. Two fixations are considered to be recurrent if they are closer than a fixed threshold $\rho$. The "Closeness" function $d$ can be defined in several ways; It can be Euclidean distance or use a grid similar to Levenshtein or ScanMatch and fixations which fall on the same AOIs will be considered close. The recurrence matrix is presumed to be diagonal. If the length of scanpaths are not identical, one of them will be truncated to have the same length (N) (Fig. 2).

$r_{P, Q}(i, j)= \begin{cases}1 & d(P[i], Q[j])<\rho \\ 0 & \text { otherwise }\end{cases}$

Let $\mathrm{C}$ be the sum of recurrences, i.e., $C=\sum_{i=1}^{N} \sum_{j=1}^{N} r_{i j}$. Further, let $D_{L}$ be the set of diagonal, $H_{L}$ the set of horizontal, and $V_{L}$ the set of vertical lines in the crossrecurrence matrix, all with a length of at least $\mathrm{L}$, and let |.| denotes cardinality. Therefore: The cross-recurrence (REC) measures the percentage of fixations that match (are close) between the two fixation sequences.

$D_{R E C}(P, Q)=100 * \frac{C}{N^{2}}$

The determinism (DET) measures the percentage of cross-recurrent points that form diagonal lines and represents the percentage of fixation trajectories common to both fixation sequences. That is, it quantifies the overlap of a specific sequence of fixations, preserving the sequential information. The minimum line length of diagonal line elements was set to $L=2$. Similarly, the laminarity (LAM) represents locations that were fixated in detail in one of the fixation sequences, but only fixated briefly in the other fixation sequence $(\mathrm{L}=2)$. Finally, the center of recurrence mass (CORM) is defined as the distance of the center of gravity from the main diagonal, normalized such that the maximum possible value is 100 . The CORM measure indicates the dominant lag of cross recurrences. Small CORM values indicate that the same fixations in both fixation sequences tend to occur close in time, whereas large CORM values indicate that cross-recurrences tend to occur with either a large positive or negative lag. Anderson et al. (2015)

$D_{D E T}(P, Q)=100 * \frac{\left|D_{L}\right|}{C}$

$D_{L A M}(P, Q)=100 * \frac{\left|H_{L}\right|+\left|V_{L}\right|}{2 * C}$

$D_{C O R}(P, Q)=100 * \frac{\sum_{i=1}^{\min (M, N)} \sum_{j=1}^{\min (M, N)}\left((j-i) r_{i j}\right.}{(\min (M, N)-1) C}$

Throughout this paper, we assumed $\rho$ is 48 equal to two degrees of visual angle (24).

\section{What can a similarity measure Do?}

Scanpath similarity measures can be used as a starting point for many more complex analyses. Collecting large amounts of eye-tracking data is easy and is getting easier, but analyzing the data in an appropriate way with the increasing wealth of information is not. It's important to develop new and powerful tools for the analysis of eye movement data. Similarity metrics can be used as a starting point for more complex analyses and developing and testing models. When it comes to similarity measures in a freeviewing task, two major approaches have normally been taken that are for model evaluation and data analysis but for different purposes. As discussed in the later part of this paper, Saccadic models exist that aim to replicate human scanpaths. Humans exhibit complex behaviors when viewing an image, which all factor into observed position, order, shape, and duration of fixations and saccades. As alluded to in the later parts of this paper, this can relate to personal characteristics, or alternatively, depend on the properties of stimuli (e.g., what's in the image). A similarity metric should be able to weigh differences and similarities among models, and in particular to differentiate and rank the models or more generally, to provide a clear measure of scanpath similarity. From a different perspective, a good similarity measure should help answer a specific but common class of questions. As noted by Mathôt et al. (2012), "it allows you to cluster similar eye movement sequences together, or detect differences between predefined groups of eye movement sequences". Cristino et al. considered four common scenarios for experiments:

1. Detecting differences between predefined sets: Given sets of eye-tracking data of recorded subjects with different conditions, the similarity measure should help to determine the effect of manipulating conditions of 
the experiment. To answer this question, normally the average similarity of pairs of eye movement sequences within sets (defined by an experimental condition) should be larger than the similarity of sequence pairs between sets.

2. Supporting scanpath theory: A similarity measure should also evaluate whether two sequences of viewing that belong to the same person (encoding and recognition) viewing the same scene (effect of person) are on average more similar than two sequences of the same person viewing different scenes (effect of image) and two sequences of different people viewing the same scene.

3. Diagnostic use: Given known groups of data, a suitably designed similarity measure (or metric) should be able to assign exemplars from a new set of eye-tracking data to known categories based on similarities. For instance, eye-tracking has been used to diagnose Alzheimer's (Biondi et al., 2017) and works reasonably well as a diagnostic tool.

4. Data-driven clustering: With a large set of data, a metric should differentiate well enough so that clustering techniques should be able to find similar clusters in data. This might comprise different people with similar viewing strategies, the same person viewing different content, different individuals viewing a common image or other explicit or implicit groupings.

\section{What makes a metric suitable?}

It is difficult to assess the value of metrics without an associated reference frame. Some studies have produced models that reveal no significant difference when compared with human data. On the basis of this, there is the temptation to assume that the model is successfully replicating human gaze patterns. However, this can be equally the success of a model, or failure of a metric. Moreover, success at discriminating between different models according to performance may be strong but lack specificity. One example of this is analysis that is purely spatial (e.g., static AUC). Another example is metrics that consider only summary statistics of gaze patterns like MultiMatch.

Given this context, we can state some guiding principles (axioms) that should inform the choice of a suitable metric. Specifically, the metric should:

1. Be capable of measuring distances between sequences of fixations.

2. Have an intuitive interpretation.

3. Be able to effectively capture the order, position, duration of fixations and shape of a scanpath (Dewhurst et al., 2012).
4. Provide a level of sensitivity that allows for reasonable separation of models that produce good vs. poor sequences

5. Should not consist only of coarse-grained saccade or fixation statistics

Each of these can be considered both in the context of the guiding principles proposed by Cristino et al. (Mathôt et al., 2012).

\section{What is missing in scanpath comparison?}

While the problem of saliency prediction has been studied in some detail, the overwhelming majority of work in this domain considers a static problem wherein the distribution of gaze, attention or salience is marked by its spatial distribution. However, spatial selection by an attentive mechanism is inherently a sequential sampling process in humans and many artificial vision systems. This evidently gains additional importance as sequential attention mechanisms in artificial vision systems are becoming increasingly prevalent.

There may be a strong bias to examine certain parts of a scene right away before moving on to other interesting regions. Alternatively, there may be several equally interesting regions of a scene and no particular order. This is a characteristic that most modeling and analysis to date has failed to address in detail. While some efforts have been devoted to analyzing sequential models of fixation, the case may be made that existing metrics fall short in adequately capturing model performance, and similarities and differences among sequences of selected regions.

Table 1 shows 18 metrics for comparing scanpaths. This clearly illustrates the amount of interest in the subject but strangely shows the lack of exploration at the meta-level in the value of metrics for analyzing behaviors and outlining strengths and weakness of these metrics. In many cases, studies that used eye-tracking data, each introduced their own metric for analyzing the data (perhaps in support of showing a desired result) but little is known about the interpretation of their metrics.

Motivated by the aforementioned observations, we revisit the space of metrics currently used in this domain to arrive at a consensus on intuitive interpretations of inter-sequence distances, and also towards redefining metrics that produce meaningful and significant contrast among observations. What follows in this paper is a presentation of experiments that ultimately advocate for an alternative metric to any appearing in the literature, ScanPath plausibility (SPP). While this is a novel metric, the essence of SPP builds on existing metrics albeit with some additional intuition related 
to the guiding principles established above. This is tested in considering a wide variety of extant saliency models coupled with a selection mechanism.

\section{How do metrics measure up?}

As alluded to earlier in this paper, a wide variety of different metrics have been proposed, and yet it is unclear the extent to which these are suitable for measuring similarity among sequences of gaze points. While many of these are grounded in measuring distances between trajectories or in computational geometry, there is a relative lack of domain knowledge inserted into the choice and use of such metrics. We therefore consider an alternative approach, and present careful analysis of information carried by different metrics and means of determining which are most discriminative. We have used various datasets for the analysis but most of the numbers are based on OSIE (Xu et al., 2014). In OSIE, eye movements are recorded from 15 users viewing 700 images of diverse everyday scenes with many semantic objects included using an EyeLink 1000 tracker at $2000 \mathrm{~Hz}$ for duration of $3 \mathrm{~s}$. Also, we have confirmed similar results on CROWD (Jiang et al., 2014) and CAT2000 (Borji \& Itti, 2015) datasets.

\section{Scanpath plausibility: Towards a more intuitive interpretation}

Consider a scene with $k$ regions that tend to be gazed at. There are several possibilities in this scenario; it is possible that some of the regions/objects are gazed at preferentially and tend to be visited in some particular order. In the extreme case, this order may be random. At the other extreme, every observer might assume the same specific order. The reality for most images is likely to be somewhere intermediate to this. E.g. Most observers might first fixate one item before shifting to a second with equal probability and some preference. Alternatively, there may be a rank ordering built into selections that are made coupled with some randomness in this order. All of these considerations present challenges for metrics. Moreover, as an image becomes more complex and contains more regions that tend to be focal points, combinatorics may imply an explosion in the degree of variability among observers (given a fixed number of focal points, there is a combinatorial explosion of sequences as the number of focal points increases) even if the amount of randomness is small.

It is a common practice to benchmark a saccadic model by averaging the distance of reproduced scanpath with scanpaths from real observers. If $\mathrm{N}$ observers produce $\mathrm{S}$, set of $\mathrm{N}$ scanpaths $s_{1}, s_{2}, . ., s_{N}$ that are in reality $\approx K(K \leq N)$ different strategies for viewing an image, and a model (x) is successful in reproducing one of these strategies $\left(s_{x}\right)$, this still implies that $\frac{K-1}{K}$ of instances included in the comparison are in some sense noise. It is evident from this line of thinking that calculating the mean of the distance score of reproduced scanpath with all other scanpaths from observers may be inappropriate. The nature of the problem when trying to predict mean scanpath similarity (MSS) behavior inherently struggles with the problem of plausible patterns being drowned out by the large number of equally plausible alternatives. With this in mind, we propose a set of similarity measures deemed ScanPath plausibility (SPP). For a given scanpath similarity measure (M), the SPP of reproduced scanpath $S_{x}$ from model (x) can be calculated using:

$\operatorname{MSS}_{M}\left(s_{x}\right)=\operatorname{Mean}_{\forall s \in S} \mathbf{M}\left(S_{x}, s\right)$

$S P P_{M}\left(s_{x}\right)=\operatorname{Min}_{\forall s \in S} \mathbf{M}\left(S_{x}, s\right)$

The heart of SPP is to measure the minimum similarity between a model's output sequence, and the set of observer scanpaths. This may be coupled with any similarity measure, but we specifically propose a few appealing options $D T W_{S P P}$, and $T D E_{S P P}$ for reasons discussed in the balance of this paper with particular emphasis placed on $D T W_{S P P}$. While a "new" metric is introduced, this is more so a different way of interpreting the (dis)similarity shown by existing metrics in considering a minimum rather than average distance from reference scanpaths. This is in recognition of the fact that several different focal points might elicit different scanpaths from observers while being variations on ordering of visiting the same set of focal points. The SPP measure is a useful adjunct measure to determine whether a scanpath is plausible on the basis of observed scanpaths rather than an average that is highly sensitive to scene complexity and number of focal points and associated combinatorics.

\section{Imposters and noise handling}

In order to better ground the interpretation of metrics, we have conducted experiments that consider human vs. human scanpath similarity. That is, given $n$ observers, one can perform a leave-one-out analysis, treating the instance left out as our exemplar, and using the remaining observers to predict gazed at points of the individual left out and determine a bound on expected model performance. We have carried out this experiment for the set of metrics considered measuring human vs. human similarity, and also human vs. imposter, where the imposter scanpath is a real scanpath but drawn from a different image. In this manner, we have the capacity to consider the extent to which 


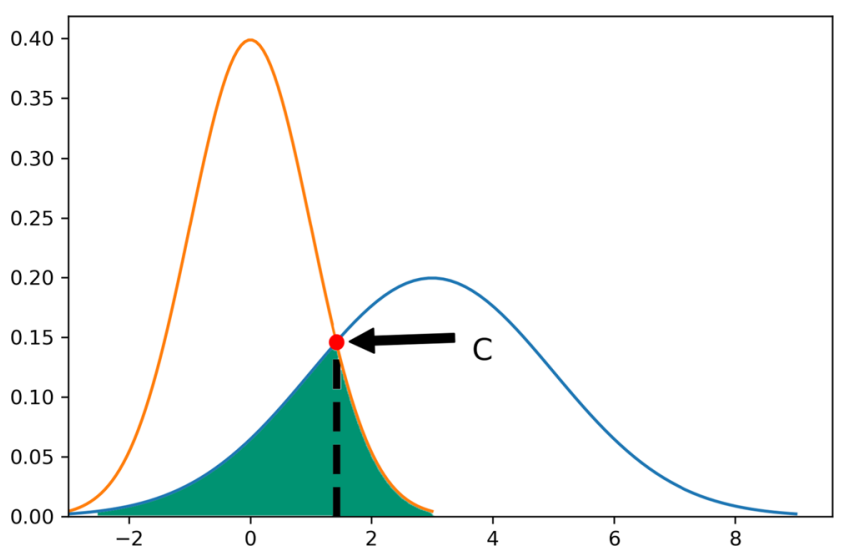

Fig. 3 Intersection of two normalized distribution. $\mathbf{C}$ is the decision theoretic criterion boundary between two normal distribution $\left(\mu_{1}=\right.$ $0, \sigma_{1}=1, \mu_{2}=3, \sigma_{2}=2$ )

scanpaths that are plausible in general conform to scanpaths that are plausible for a particular image. In practice, a good scanpath similarity measure should be capable of discerning what sequences belong to a common image and Identify those that are not specific to the image considered. For metric $\mathrm{M}$, in considering the overall distributions of scanpath similarity scores $A$ and $B$ with mean and standard deviations $\mu_{A}$ and $\sigma_{A}$, and $\mu_{B}$ and $\sigma_{B}$, respectively, where $A$ is all instances of leave-one-out comparisons from the same image, and $B$ is all instances of leave-one-out where the comparator is an imposter, we can readily calculate the overlap among the two distributions. This corresponds to the proportion of instances that belong to one distribution, but would be classified as belonging to the alternative. A lower degree of overlap is diagnostic of a more powerful metric for discerning these two groups (following classic decision theory). If $\mathrm{C}$ is presumed as the center of the intersection and $\mathrm{F}$ is the cumulative distribution function as visualized in Fig. 3, the ratio of intersection is given by:

$P\left(X_{1}>c\right)+P\left(X_{2}<c\right)=1-F_{1}(c)+F_{2}(c)$

$=1-\frac{1}{2} \operatorname{erf}\left(\frac{c-\mu_{1}}{\sqrt{2} \sigma_{1}}\right)+\frac{1}{2} \operatorname{erf}\left(\frac{c-\mu_{2}}{\sqrt{2} \sigma_{2}}\right)$

where $\mathrm{C}$ can be calculated by:

$c=\frac{\mu_{2} \sigma_{1}^{2}-\sigma_{2}\left(\mu_{1} \sigma_{2}+\sigma_{1} \sqrt{\left(\mu_{1}-\mu_{2}\right)^{2}+2\left(\sigma_{1}^{2}-\sigma_{2}^{2}\right) \log \left(\frac{\sigma_{1}}{\sigma_{2}}\right)}\right)}{\sigma_{1}^{2}-\sigma_{2}^{2}}$
This assumes that histograms must follow a normal distribution. We tried a normality test based on Agostino and Pearson's tests. The test combines skew and kurtosis to produce an omnibus test of normality and all the metrics rejected the null hypothesis. Final results are shown in Table 2. It is immediately evident that a few metrics have poor power of discrimination while others stand out. It is also the case that some that lack discriminative power fail to meet some of the considerations defined under "What makes a metric suitable?"

In addition to revealing behavior of metrics the results of this experiment for SPP are unequivocal. The SPP distances show significantly higher capacity for separating these classes in part due to resilience to domain relevant noise that is intrinsic to inter-observer variability, but also given the high intrinsic variability of scanpaths that implies a large distance when an imposter is considered.

To take this analysis further, we ask a different question. Suppose inter-observer scanpath similarity is considered for a given image, and this is compared with interobserver scanpath similarity where an increasing number of imposters is introduced to the observer pool. In this case, leave one out analysis is performed on the group for all $\mathrm{N}$ observers with $\mathrm{K}$ imposters $(\mathrm{K}<\mathrm{N}-1)$. One can also consider how the distributions diverge as the observer pool is increasingly polluted with random scanpaths from other images. The results of this analysis appear in Fig. 4. This analysis also reveals differences among the base metrics in their discriminative power.

One can see that the SPP based on a min distance across observers is much less sensitive to noisy samples being introduced. Moreover, when the sample is entirely or almost entirely imposters, the distributions become very distinct. On the other hand, the standard metrics show a monotonic and linear drop-off revealing the sensitivity to noise and also again hinting at weaker discrimination in the space of comparing scanpaths. This analysis also reveals differences among the base metrics in their discriminative power.

\section{Robustness to issues in scanpath comparison}

Taking a principled approach, one may consider various types of perturbations that can occur from one scanpath

Table 2 Area of intersection for two distributions based on leave one out analysis. One is mean of inter-observer similarities while viewing the same image, and the other is between images or when only imposter samples exist in the pool similar to Fig. 4. In practice, a strong metric should elicit a very different distribution

\begin{tabular}{llllllllllllllllll}
\hline & EUC & MAN & EYE & LEV & SMT & HAU & FRE & DTW & TDE & REC & DET & LAM & CORM & MM_S & MM_A & MM_L & MM_P \\
\hline MSS & 0.82 & 1.00 & 0.29 & 0.61 & 1.00 & 0.71 & 0.90 & 0.60 & 0.58 & 1.00 & 0.75 & 1.00 & 0.90 & 1.00 & 1.00 & 1.00 & 1.00 \\
SPP & 0.67 & 1.00 & 0.15 & 0.49 & 1.00 & 0.46 & 0.71 & 0.37 & 0.46 & 1.00 & 0.46 & 1.00 & 1.00 & 1.00 & 0.98 & 1.00 & 1.00 \\
\hline
\end{tabular}




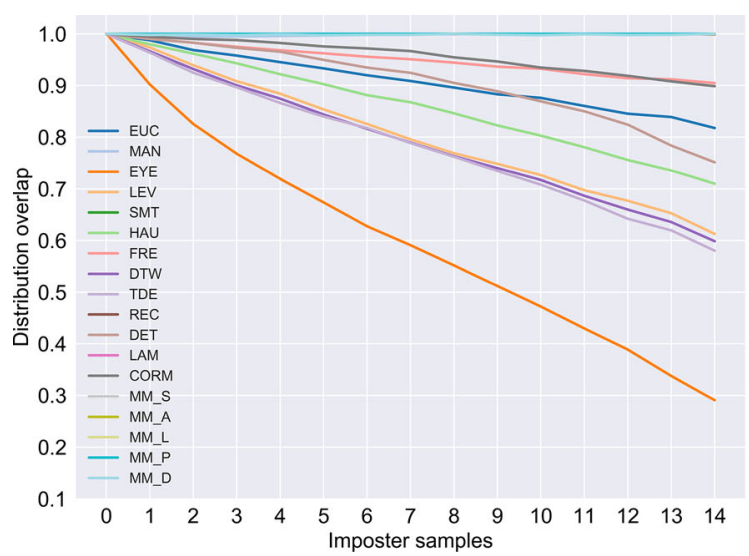

Fig. 4 Sensitivity to pollution of the observer pool by an increasing number of imposter samples. This effectively measures inter-observer similarity for a pool of $N=15$ observers per image where $k=[0-14]$ are sequences chosen at random from observers of a different image.

to another. In a natural setting, one might expect these differences to be predominately driven by differences among viewing patterns across observer or from one image to another. However, the discernibility of differences can be studied in the context of a family of specific perturbations. It is worth noting that some of these perturbations may also occur by virtue of noise in measurement of gaze position, or by virtue of the locally stochastic nature of gaze points.

A set of possible perturbations is shown in Fig. 5. This figure reveals different perturbations of a scanpath that should result in differences in similarity scores, but also provides significant insight into the degree of sensitivity of different measurements to these perturbations. These are as follows:

1. Spatial noise: The individual fixation locations may be perturbed spatially and independently, while maintaining their overall order. The noise is a random sample from a Gaussian distribution with $\sigma$ according to degree of visual angle.

2. Spatial offset: Individual fixation locations may be perturbed in a common fashion, where each is subject to the same spatial transformation (2D translation). The offset is a random sample from a Gaussian distribution with $\sigma$ according to degree of visual angle.

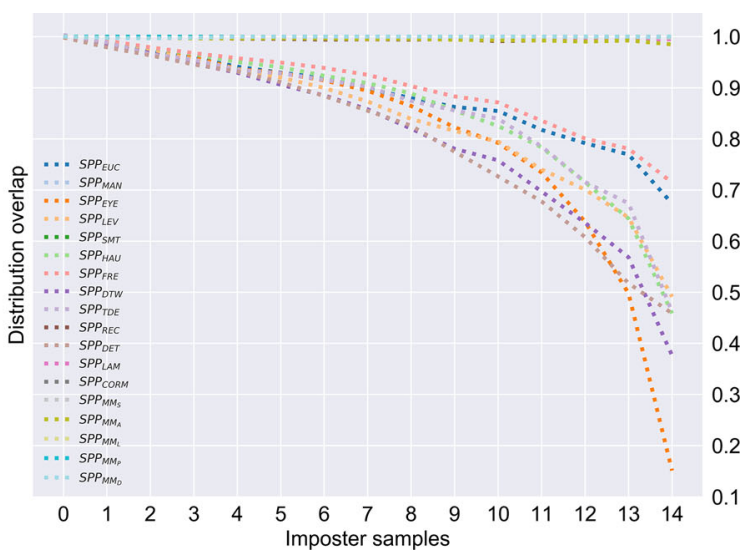

$k$ is gradually increased to examine the degree of sensitivity to noise samples. Solid lines are MSS and dashed lines are SPP measures. Points are slightly jittered to avoid overlapping

3. Ordinal offset: The specific ordering may be offset. (e.g., supposing two sequences are identical, but one subsequence starts at fixation $\mathrm{N}$ and the other at fixation $\mathrm{N}+1$ with equal lengths. At step 0 , each scanpath is being compared with itself.

4. Reversed: All fixation locations are identical, but the order they are visited in is reversed. At step 0 , each scanpath is being compared with the exact opposite ordering of itself.

5. Scaled: The overall geometry of the fixation patterns is identical, but one of the two is subject to global spatial scaling so that the degree of eccentricity of fixations is smaller or larger in the scaled case.

We take each of these perturbations and gradually add an increasing level of noise to scanpaths. Each time, we calculate the similarity of the noisy scanpath with the base (untouched) scanpath. Figures 6, 7, 8 and 9 shows the mean of similarities/dissimilarities among all samples in the dataset at different noise levels. In all of these instances, a metric should be capable of discerning differences, albeit the point of interpretability can be understood much more clearly in the context of observing the degree of sensitivity to such perturbations. In this regard, we observe the following:
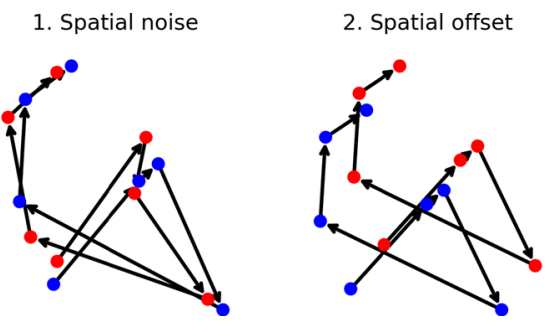

Fig. 5 A depiction of different conditions that may reveal sensitivity of sequential metrics. These may happen by virtue of small differences in viewing patterns, noise in data capture, or the overall stochastic nature
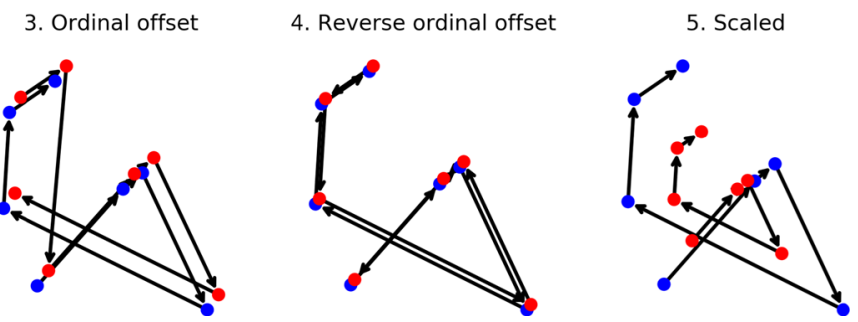

of the process. The blue scanpath is the reference sequence and the red scanpath is the transformed scanpath. (Derived from Dewhurst et al. (2012)) 

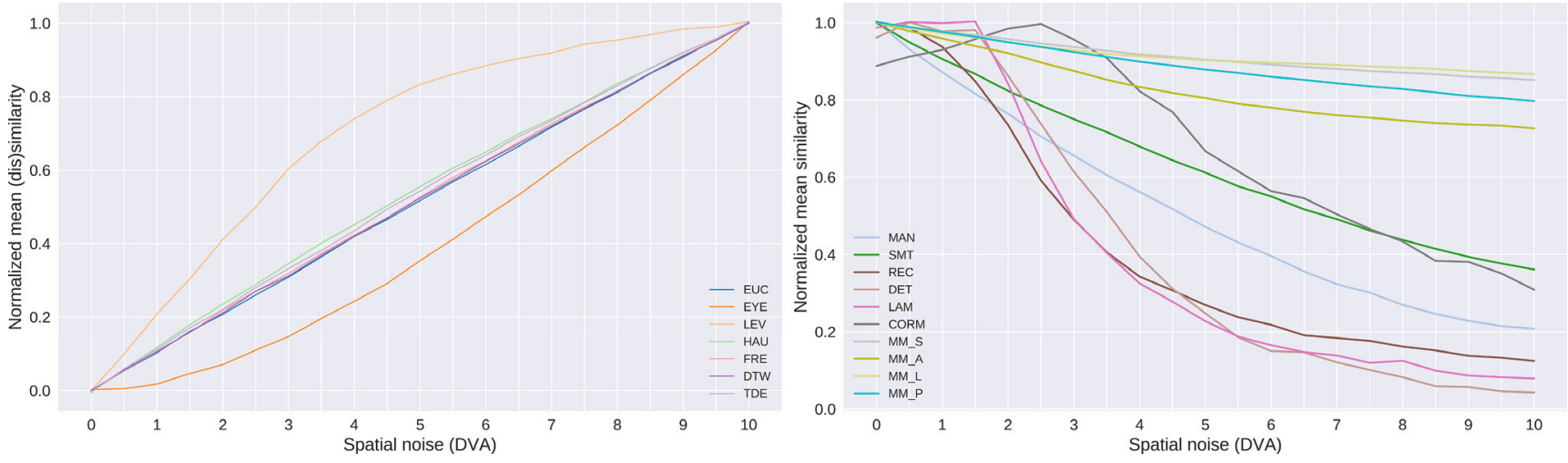

Fig. 6 Spatial noise: Each fixation has been moved according to a random sample from a Gaussian distribution with $\sigma$ according to degree of visual angle. This plot is normalized by maximum value of each metric. Please refer to Appendix for actual values and error bars

Figure 6 reveals the sensitivity of different similarity measures to the addition of spatial noise. There is a notable dropoff for increasing spatial offset, that is linear in most instances for the ScanMatch and MultiMatch metrics; some similarity measures show less sensitivity to spatial offset. For other measures, the behavior is much more mixed. A family of dis-similarity measures in Fig. 7 show common patterns in their behavior as spatial offset is increased, the average distance produced by the models increases monotonically. Also, some models fail to adhere to this behavior. For the case of "tied" spatial offset in Fig. 7 where all points are subject to a common transformation, the family of metrics that are well behaved for perturbations show the same well-behaved monotonic increase in interscanpath distance. overall spatial offset shows similar effect as spatial noise.

Figure 8 depicts sensitivity to ordinal offset. As the degree of ordinal offset increases, one observes a decrease for the ScanMatch and MultiMatch metrics and virtually no change for SMT, HAU and EYE metrics. In the case of decrease, this follows an inverse sigmoid profile. For other metrics (in particular those that are well behaved with respect to spatial perturbations), there is consistency in an increase in distance subject to spatial offset, which is followed by a decrease for larger ordinal offsets. Note that this is a byproduct of the number of sequences that are sufficiently long to allow such an ordinal offset to be considered, which is much higher for five than for any of the cases beyond this. The reversed ordinal offset shown in Fig. 9 demonstrates sensitivity to distance subject to a reversal of the sequence. In this case, virtually all of the metrics show a relatively small degree of sensitivity to reversal albeit this becomes quite pronounced for the higher reverse ordinal offset.

Overall, this analysis reveals some interesting characteristics of the sensitivity of distances to different types of perturbations. In some instances, scores are quite erratic, or vary in a manner that is counterintuitive. In other cases, models appear well behaved, and we also seem some degree of correlation appearing at a qualitative level among how these distances vary as the degree of correlation is varied. Models that seem to show the most sensitivity include EYE, DTW, DET, and to a lesser extent LEV, HAU, TDE. However, EYE and DET seem to show a weakness to ordinal offset, the former by construction.
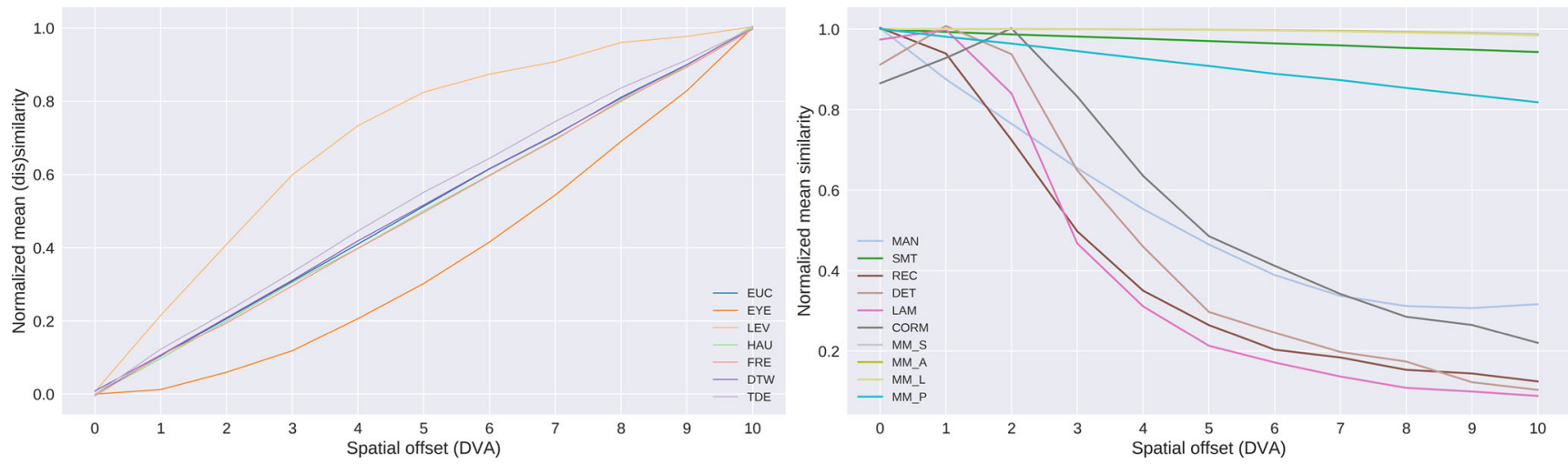

Fig. 7 Spatial offset: All fixations have been moved according to a random sample from a Gaussian distribution with $\sigma$ according to degree of visual angle. This plot is normalized by maximum value of each metric. please refer to Appendix for actual values and error bars 

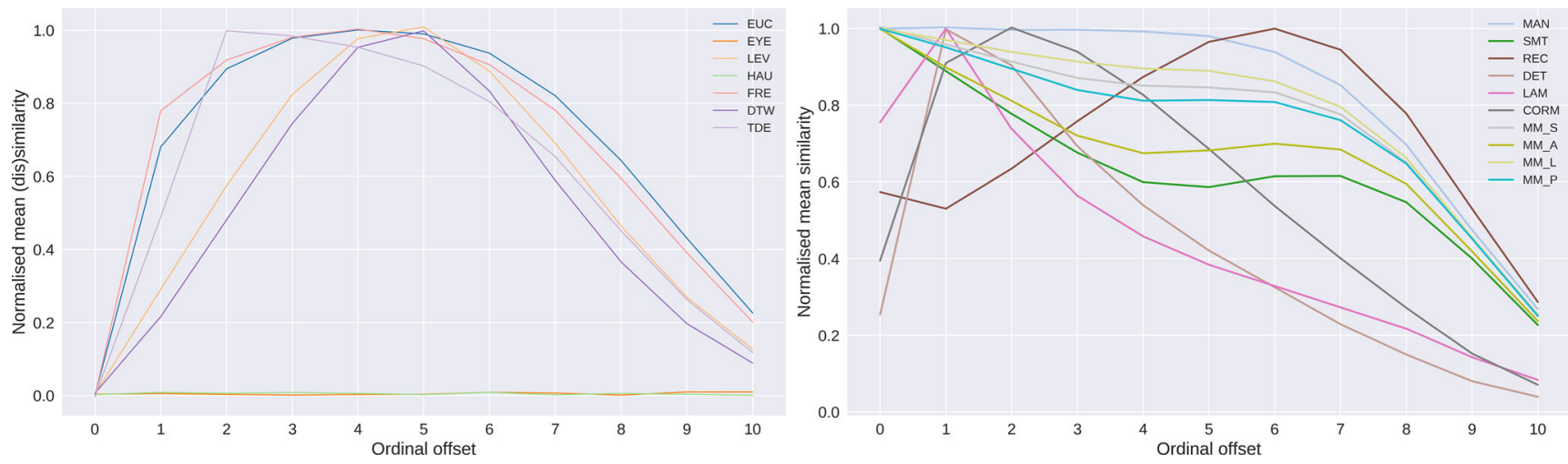

Fig. 8 Ordinal offset: Average distance when scanpaths have temporally shifted in a clockwise direction. This plot is normalized by maximum value of each metric. please refer to Appendix for actual values and error bars

It is also worth mentioning that the combination of variation of (dis)similarity measures subject to imposter scanpaths introduced, and sensitivity to various types of scanpath noise allow for something to be discerned about sensitivity to scanpath distances versus sensitivity to inherent noise in fixations captured. The noise inherent in scanpaths from the eye tracker itself will cause measures to show greater similarity or dissimilarity. For a large enough pool of observers, the SPP measures help to drown out the effects of imprecision (noise) in the raw data captured in so far as metric sensitivity is concerned.

\section{What is captured by different metrics?}

Most similarity measures proposed have a history that derives from measuring similarity of trajectories, or have roots in sequential comparisons which tend to imply metrics that cast trajectories as strings. While some comparisons have been made among such metrics (Anderson et al., 2015), there is not yet a consensus on what makes for a suitable metric. Moreover, little attention has been paid to the intuitive interpretation of these metrics or their qualitative behavior.
Figure 11 shows for a variety of metrics a reference scanpath in green, and a number of sequences from other observers ranked from most similar to least similar (left to right) each according to a specific metric. The specific metrics shown are referenced in the figure caption. As this figure reveals, there is some intuitive sense that at least a subset of these metrics is representative of the degree of agreement between pairs of scanpaths. It is not clear however how to discern which metric is most useful. Moreover, it can be seen that scanpaths can be highly divergent even if there is general agreement on a few items in the scene lending support to the argument put forth for metrics that capture scanpath plausibility (SPP).

Given that there are a variety of metrics, one might also surmise that some of these may carry similar information, while others may differ significantly in their characterization of distance. One way of considering this directly is by examining the correlation structure among different metrics across a wide array of saliency models and images to determine which produce similar model rankings, or more specifically, to what extent is the structure of relative model rankings similar. To this end, we examine a variety of different saliency algorithms and quantify
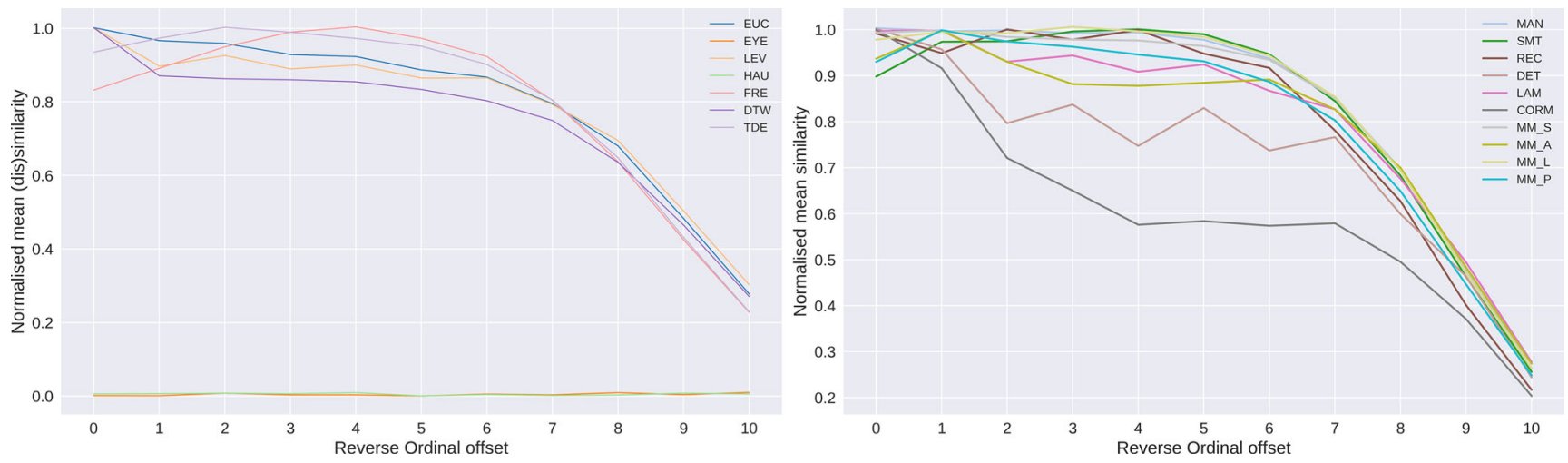

Fig. 9 Reverse Ordinal offset: Average distance when scanpaths have been temporally shifted in a counter-clockwise direction. This plot is normalized by maximum value of each metric. please refer to Appendix for actual values and error bars 
the Pearson correlation among different metrics across algorithms. This analysis appears in Fig. 10.

Note that some metrics (e.g., Levenshtein distance) are poorly correlated with others, while others show a greater degree of agreement. With that said, it remains unclear what power these metrics carry in quantifying how well a pair of scanpaths match or how well a predicted scanpath matches a set of ground truth exemplars (Fig. 11).

\section{Is SPP more useful in determining model performance?}

In the "How do metrics measure up?", a decision theoretic definition was put forth for measuring how discriminative two models are with respect to a metric. The crux of this, is that if a metric is applied to two different populations of data (e.g., viewers of the same image vs. different images) each of these implies a distribution of distances for the withinimage and different-image comparison. For example, if N-1 observers are measured in their distance to 1 observer $\mathrm{N}$ times in a leave one out fashion, this can be done for either the 1 left out, or a random imposter from another image. In this manner, we are able to measure the extent to which a metric can discern whether two viewers examined the same image or different images. Results from this experiment for SPP-based scanpath measures are also shown in Fig. 4. The area of overlap between these two distributions is a useful

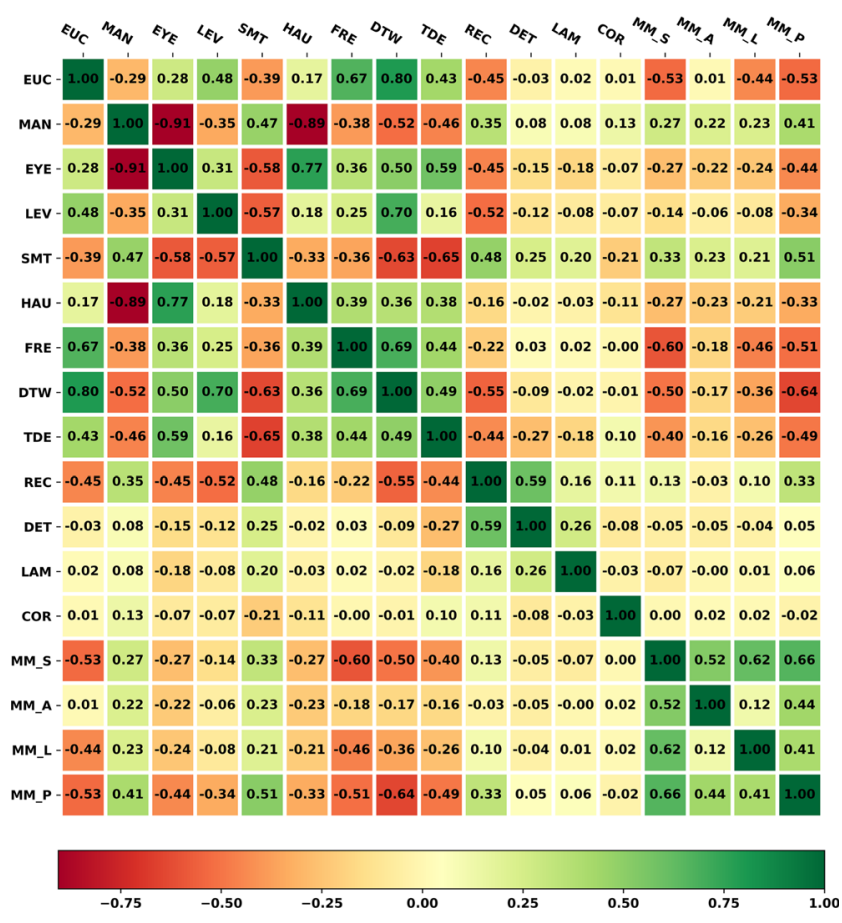

Fig. 10 The Spearman rank correlation among different metrics across algorithms measure of the degree of confusion one might expect, where 0 implies no overlap among distances. In this instance, it is clear that there is a significant advantage to some metrics, but also to the SPP variant in particular.

In addition to revealing behavior of metrics the results of this experiment for SPP are unequivocal. The SPP measures show significantly higher capacity for separating these classes in part due to resilience to domain relevant noise that is intrinsic to inter-observer variability, but also given the high intrinsic variability of scanpaths that implies a large distance when an imposter is considered. In Fig. 4, One can see that the SPP based on a min distance across observers is much less sensitive to noisy samples being introduced. Moreover, when the sample is entirely or almost entirely imposters as in Table 2, the distributions become very distinct. Alternatively, the standard metrics show a monotonic and somewhat linear drop-off revealing the sensitivity to noise and also again hinting at weaker discrimination in the space of comparing scanpaths.

This confirms the observation that more separation can be observed based on $S P P$, and that traditional metrics would lead one to conclude that the 2 sets of observations are identical for many metrics. At first glance, one might be inclined to rely on the EYE metric for its separability and behavior as imposters are introduced. However, it is notable that ordinal perturbations are not considered in this metric. For this reason, one might consider using both $D T W_{S P P}$ and $E Y E$ in combination to measure model performance or compare populations of observers.

\section{Summary}

In this paper, we have examined the problem of scanpath prediction with an emphasis on suitable metrics for comparing scanpaths. we have shown how metrics that have traditionally been used in this domain lack an intuitive interpretation, and also provide weak contrast for revealing differences in model capabilities. We have provided careful analysis of different metrics and their characteristics and proposed a viable alternative that provides a stronger measure for model-human or model-model distances while also revealing which existing metrics provide the strongest power of discrimination. Finally, we provide some specific recommendations for metrics and considerations that are important in moving forward.

\section{Codes and data}

The data and materials for all experiments will be available at https://github.com/rAm1n/saliency 

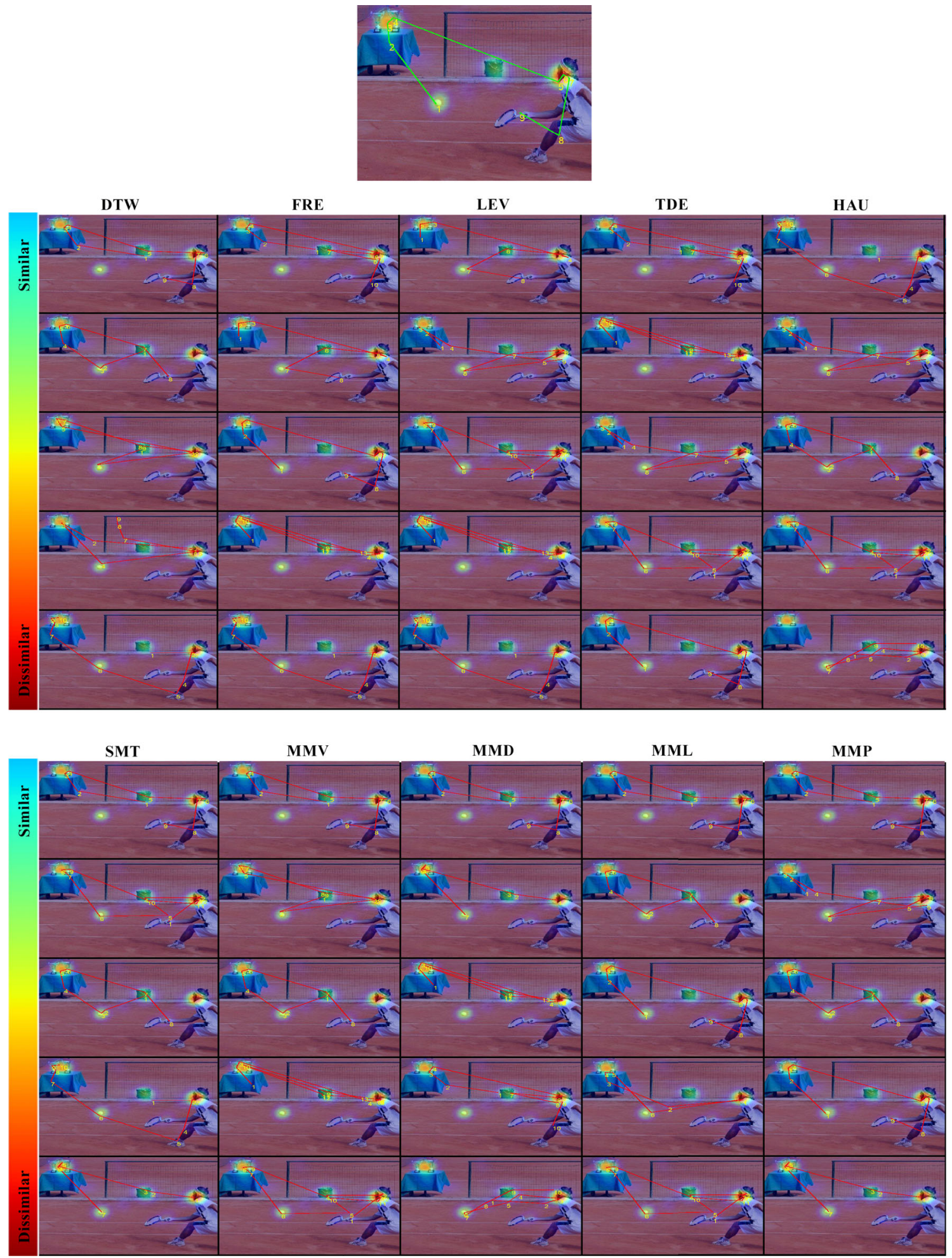

Fig. 11 Comparison between a reference scanpath (green) and scanpaths from other observers (red). Observer scanpaths are shown based on degree of similarity to the reference (top: most similar to bottom: least similar). Each column corresponds to a different metric that characterizes the degree of similarity as labeled 


\section{Appendix}
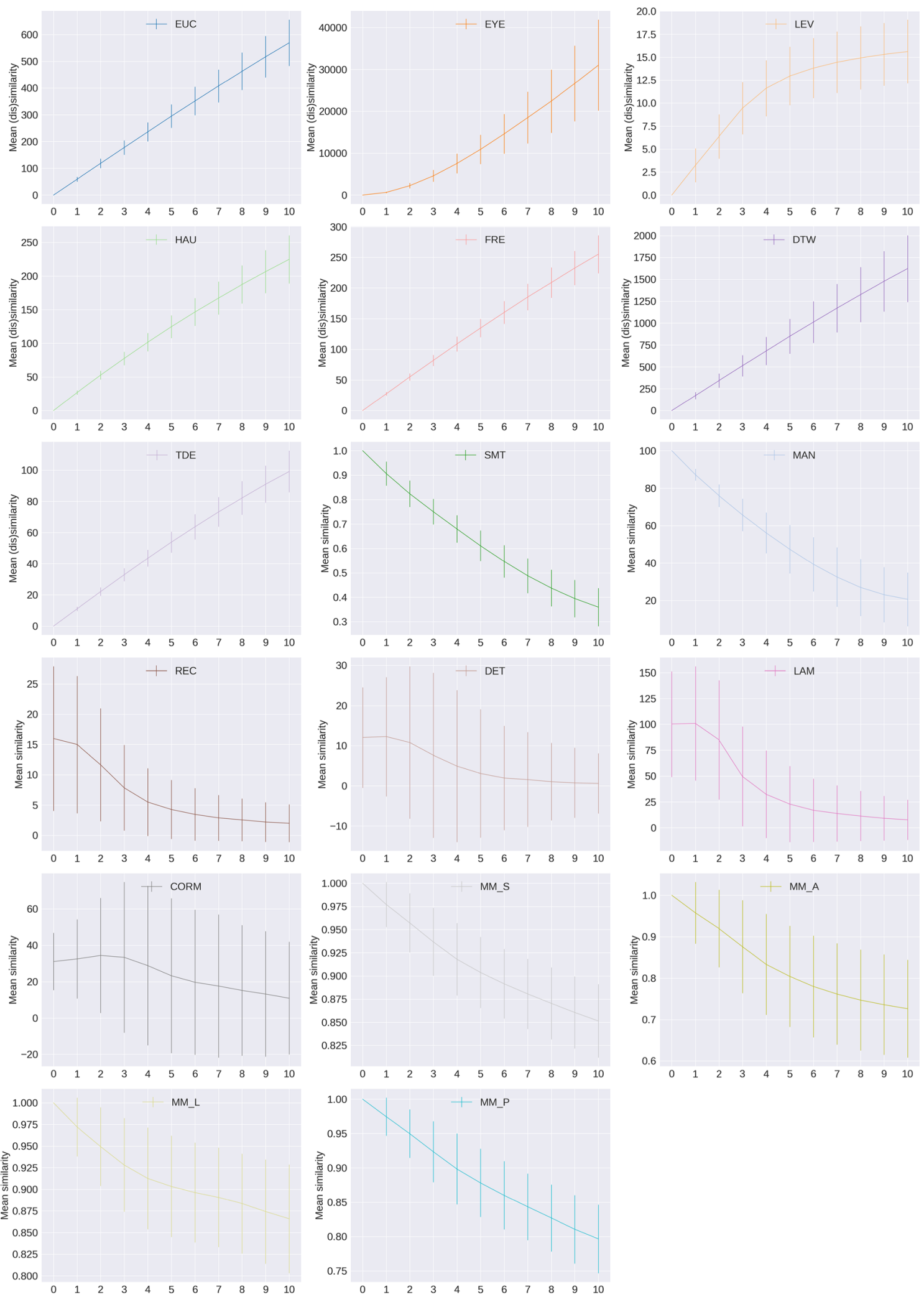

Fig. 12 Standard deviation error bars and distribution of data for spatial noise experiment in Fig. 6 

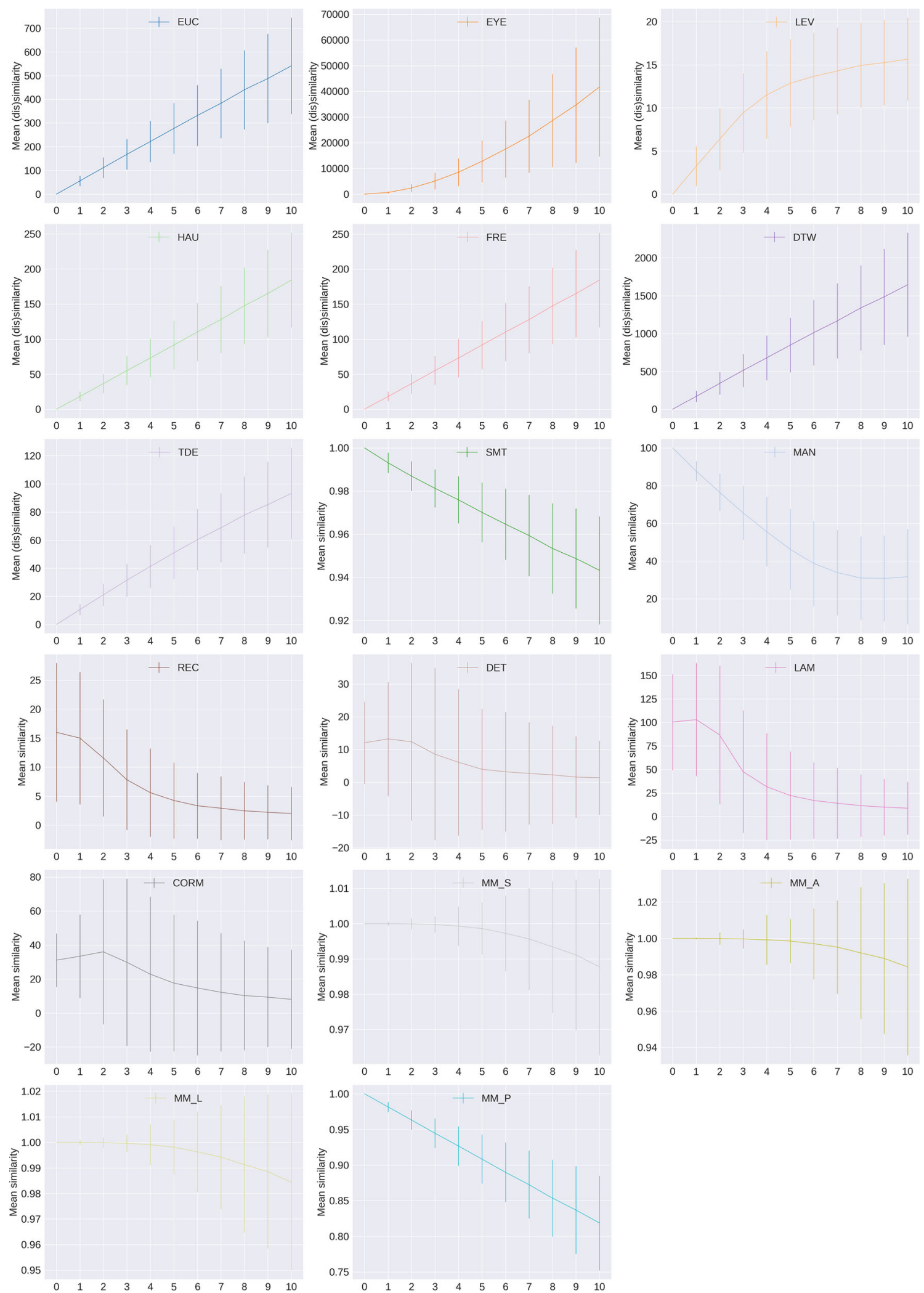

Fig. 13 Standard deviation error bars and distribution of data for spatial offset experiment in Fig. 7 

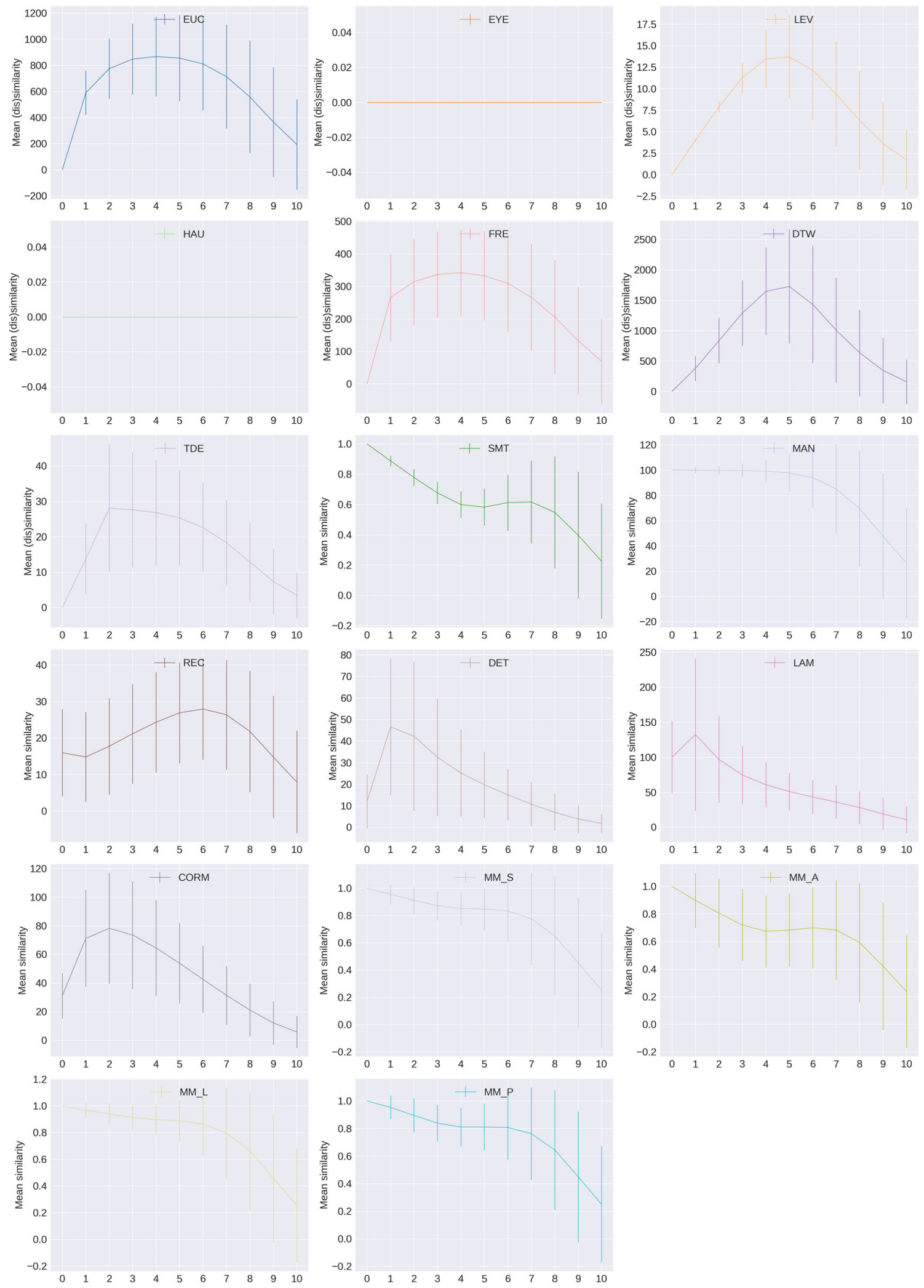

Fig. 14 Standard deviation error bars and distribution of data for ordinal offset experiment in Fig. 8 

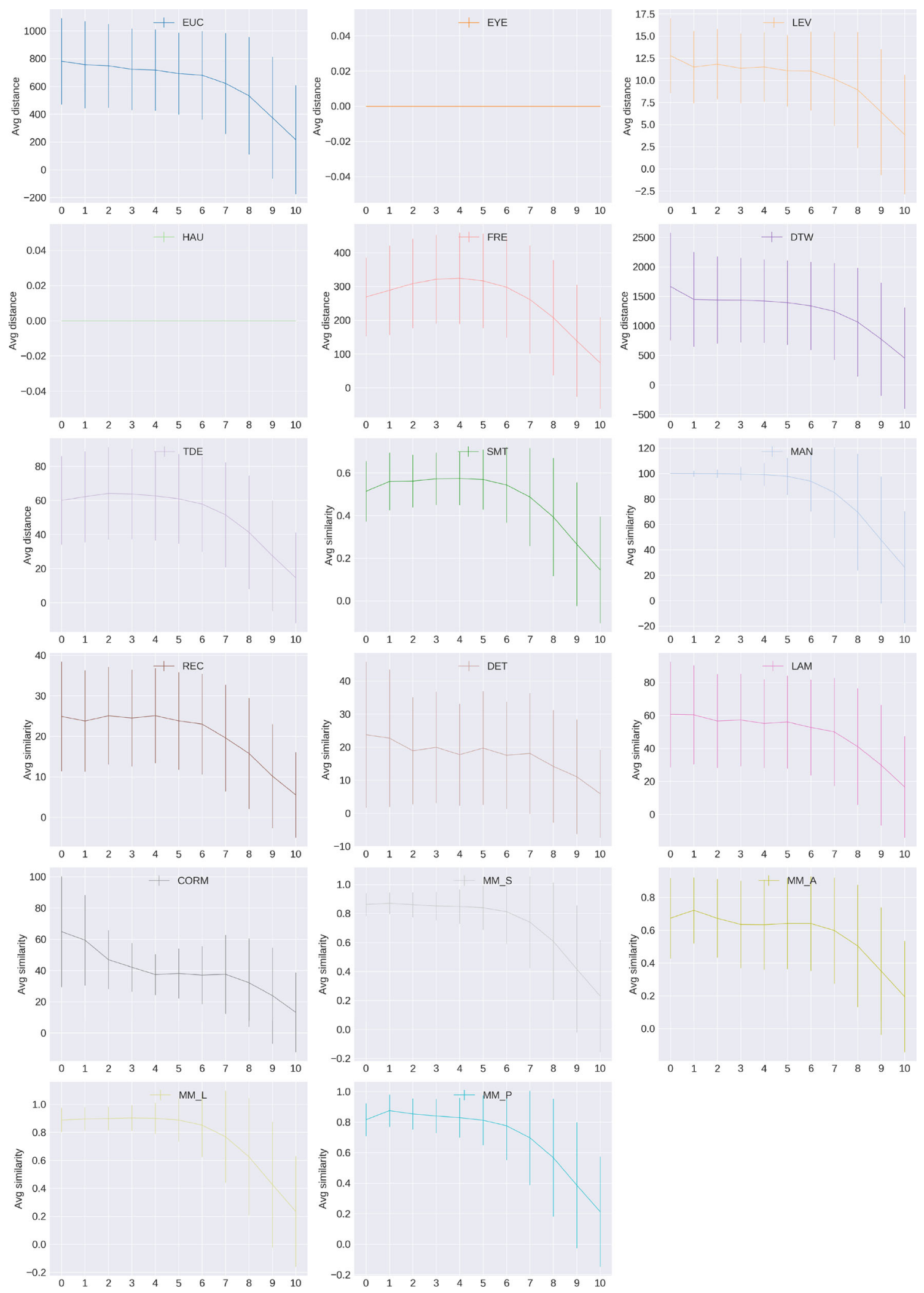

Fig. 15 Standard deviation error bars and distribution of data for reverse ordinal offset experiment in Fig. 9 


\section{References}

Adeli, H., \& Zelinsky, G. (2018). Deep-BXN: Deep networks meet biased competition to create a brain-inspired model of attention control. In Proceedings of the IEEE conference on computer vision and pattern recognition workshops, (pp. 1932-1942).

Anderson, N. C., Anderson, F., Kingstone, A., \& Bischof, W. F. (2015). A comparison of scanpath comparison methods. Behavior Research Methods, 47(4), 1377-1392.

Anderson, N. C., Bischof, W. F., Laidlaw, K. E., Risko, E. F., \& Kingstone, A. (2013). Recurrence quantification analysis of eye movements. Behavior Research Methods, 45(3), 842856.

Anderson, N. C., Bischof, W. F., Laidlaw, K. E. W., Risko, E. F., \& Kingstone, A. (2013). Recurrence quantification analysis of eye movements. Behavior Research Methods, 45(3), 842-856.

Aronov, B., Har-Peled, S., Knauer, C., Wang, Y., \& Wenk, C. (2006). Fréchet distance for curves, revisited. In European symposium on algorithms, (pp. 52-63): Springer.

Berndt, D. J. (1994). Using dynamic time warping to find patterns in time series. In KDD workshop, (Vol. 10, pp. 359-370). WA: Seattle.

Biondi, J., Fernandez, G., Castro, S., \& Agamennoni, O. (2017). Eye-movement behavior identification for ad diagnosis. arXiv:1702.00837.

Borji, A., \& Itti, L. (2015). Cat2000:, A large-scale fixation dataset for boosting saliency research. arXiv:1505.03581.

Bruce, N., Catton, C., \& Janjic, S. (2016). A deeper look at saliency:, Feature contrast, semantics, and beyond, pp. 516-524.

Cristino, F., Mathôt, S., Theeuwes, J., \& Gilchrist, I. D. (2010). Scanmatch: a novel method for comparing fixation sequences. Behavior Research Methods, 42(3), 692-700.

Dewhurst, R., Nyström, M., Jarodzka, H., Foulsham, T., Johansson, R., \& Holmqvist, K. (2012). It depends on how you look at it: Scanpath comparison in multiple dimensions with multimatch, a vector-based approach. Behavior Research Methods, 44(4), 10791100 .

Duchowski, A. T., Driver, J., Jolaoso, S., Tan, W., Ramey, B. N., \& Robbins, A. (2010). Scanpath comparison revisited. In Proceedings of the 2010 symposium on eye-tracking research \& applications, (pp. 219-226): ACM.

Eiter, T., \& Mannila, H. (1994). Computing discrete Fréchet distance. Technical report, Citeseer.

Foulsham, T., Dewhurst, R., Nyström, M., Jarodzka, H., Johansson, R., Underwood, G., \& Holmqvist, K. (2012). Comparing scanpaths during scene encoding and recognition: A multi-dimensional approach. Journal of Eye Movement Research, 5(4). https://doi.org/10.16910/jemr.5.4.3, https://bop. unibe.ch/JEMR/article/view/2341.

Foulsham, T., \& Underwood, G. (2008). What can saliency models predict about eye movements? Spatial and sequential aspects of fixations during encoding and recognition. Journal of Vision, 8(2), 6-6.

Henderson, J. M., Brockmole, J. R., Castelhano, M. S., \& Mack, M. (2007). Visual saliency does not account for eye movements during visual search in real-world scenes. In Eye movements, (pp. 537-III): Elsevier.

Huttenlocher, D. P., Klanderman, G. A., \& Rucklidge, W. J. (1993). Comparing images using the hausdorff distance. IEEE Transactions on Pattern Analysis and Machine Intelligence, 15(9), 850-863.

Islam, M. A., Kalash, M., \& Bruce, N. D. (2018). Revisiting salient object detection: Simultaneous detection, ranking, and subitizing of multiple salient objects. In Proceedings of the IEEE international conference on computer vision.

Islam, M. A., Kalash, M., Rochan, M., Bruce, N. D., \& Wang, Y. (2017). Salient object detection using a context-aware refinement network. In British machine vision conference.

Jiang, M., Xu, J., \& Zhao, Q. (2014). Saliency in crowd. In European conference on computer vision, (pp. 17-32): Springer.

Koch, C., \& Ullman, S. (1985). Shifts in selective visual attention: towards the underlying neural circuitry. In Matters of Intelligence, (pp. 115-141): Springer.

Kuo, W., Hariharan, B., \& Malik, J. (2015). Deepbox: Learning objectness with convolutional networks. In Proceedings of the IEEE international conference on computer vision, (pp. 24792487).

Mannan, S. K., Ruddock, K. H., \& Wooding, D. S. (1996). The relationship between the locations of spatial features and those of fixations made during visual examination of briefly presented images. Spatial Vision, 10(3), 165-188.

Mathôt, S., Cristino, F., Gilchrist, I. D., \& Theeuwes, J. (2012). A simple way to estimate similarity between pairs of eye movement sequences. Journal of Eye Movement Research, 5(1).

Meur, L. (2015). Saccadic model of eye movements for free-viewing condition. Vision Research, 116, 152-164.

Needleman, S. B., \& Wunsch, C. D. (1970). A general method applicable to the search for similarities in the amino acid sequence of two proteins. Journal of Molecular Biology, 48(3), 443-453.

Noton, D., \& Stark, L. (1971). Scanpaths in eye movements during pattern perception. Science, 171(3968), 308-311.

Pellicano, E., Smith, A. D., Cristino, F., Hood, B. M., Briscoe, J., \& Gilchrist, I. D. (2011). Children with autism are neither systematic nor optimal foragers. Proceedings of the National Academy of Sciences, 108(1), 421-426.

Privitera, C. M., \& Stark, L. W. (2000). Algorithms for defining visual regions-of-interest: Comparison with eye fixations. IEEE Transactions on Pattern Analysis and Machine Intelligence, 22(9), 970-982.

Samuel, A. G., \& Kat, D. (2003). Inhibition of return: A graphical meta-analysis of its time course and an empirical test of its temporal and spatial properties. Psychonomic Bulletin \& Review, 10(4), 897-906.

Sauer, T., Yorke, J. A., \& Casdagli, M. (1991). Embedology. Journal of Statistical Physics, 65(3-4), 579-616.

Sharafi, Z., Soh, Z., \& Guéhéneuc, Y.-G. (2015). A systematic literature review on the usage of eye-tracking in software engineering. Information and Software Technology, 67, 79-107.

Tatler, B. W., Baddeley, R. J., \& Gilchrist, I. D. (2005). Visual correlates of fixation selection: Effects of scale and time. Vision Research, 45(5), 643-659.

Wang, W., Chen, C., Wang, Y., Jiang, T., Fang, F., \& Yao, Y. (2011). Simulating human saccadic scanpaths on natural images. In 2011 IEEE conference on computer vision and pattern recognition (CVPR), (pp. 441-448): IEEE.

Xu, J., Jiang, M., Wang, S., Kankanhalli, M. S., \& Zhao, Q. (2014). Predicting human gaze beyond pixels. Journal of Vision, 14(1), $28-28$.

Xu, K., Ba, J., Kiros, R., Cho, K., Courville, A., Salakhudinov, R., ..., Bengio, Y. (2015). Show, attend and tell: Neural image caption generation with visual attention. In International conference on machine learning, (pp. 2048-2057).

Publisher's note Springer Nature remains neutral with regard to jurisdictional claims in published maps and institutional affiliations. 